\title{
Téoros
}

Revue de recherche en tourisme

\section{La participation des communautés locales dans les parcs nationaux au nord du $49^{\mathrm{e}}$ parallèle}

\section{Philippe Desroches et Martine Freedman}

Volume 31, numéro 1, 2012

URI : https://id.erudit.org/iderudit/1020715ar

DOI : https://doi.org/10.7202/1020715ar

Aller au sommaire du numéro

Éditeur(s)

Université du Québec à Montréal

ISSN

0712-8657 (imprimé)

1923-2705 (numérique)

Découvrir la revue

Citer cette note

Desroches, P. \& Freedman, M. (2012). La participation des communautés locales dans les parcs nationaux au nord du $49^{\mathrm{e}}$ parallèle. Téoros, 31(1),

124-126. https://doi.org/10.7202/1020715ar d'utilisation que vous pouvez consulter en ligne. 


\title{
La participation des communautés locales dans les parcs nationaux au nord du $49^{\mathrm{e}}$ parallèle
}

\author{
Philippe DESROCHES, M.A. \\ Direction des connaissances stratégiques en tourisme \\ Tourisme Québec, ministère des Finances et de l'Économie \\ philippe.desroches@tourisme.gouv.qc.ca
}

\author{
Martine FREEDMAN, Ph.D. \\ Direction des stratégies et politiques touristiques \\ Tourisme Québec, ministère des Finances et de l'Économie \\ martine.freedman@tourisme.gouv.qc.ca
}

Depuis 2008, Tourisme Québec se penche de façon plus marquée sur le développement touristique au nord du $49^{\mathrm{e}}$ parallèle. Des parcs nationaux figurent dans l'offre actuelle et potentielle de cette région. En raison de l'ampleur de tels projets et des caractéristiques des populations de cette région, la question de la participation des communautés locales tant dans l'élaboration des parcs, dans leur mise en place que dans leur exploitation se doit d'être examinée.

Nous présenterons dans cette chronique comment les communautés locales participent et participeront à l'exploitation des parcs, plus spécifiquement pour quatre d'entre eux, soit : le parc national Kuururjuaq, le projet de parc national Tursujuq (nommé auparavant parc national des LacsGuillaume-Delisle-et-à-l'Eau-Claire), le projet de parc national Ulittaniujalik (appelé avant «des Monts-Pyramides») et le projet de parc national Albanel-Témiscamie-Otish. Notons que ces quatre parcs s'ajouteront aux parcs nationaux des Pingualuit et de la Kuururjuaq, créés respectivement en 2004 et en 2009. En d'autres termes, il s'agit d'abord de préciser ce que nous entendons par leur participation, puis de discuter comment cette participation est réalisée. Avant d'aller plus loin, il est nécessaire de rappeler quelques caractéristiques des parcs nationaux au Québec.

Un parc national a pour «objectif prioritaire d'assurer la conservation et la protection permanente de territoires représentatifs des régions naturelles du Québec ou de sites naturels à caractère exceptionnel, notamment en raison de leur diversité biologique, tout en les rendant accessibles au public pour des fins d'éducation et de récréation extensive» (Québec, 1977). Dans ce contexte, les activités traditionnelles d'exploitation de la faune et de la flore pourraient s'avérer incompatibles avec l'établissement de parcs nationaux. Toutefois, en vertu des droits dont bénéficient les populations amérindiennes et inuites (Québec, s. d.), ces dernières pourront continuer leurs activités de chasse et de pêche même sur les territoires des futurs parcs (Gouvernement du Québec, 2005). Notons que dans le projet du parc national Ulittaniujalik, si le droit de se livrer à des activités d'exploitation traditionnelles par les Inuits n'est pas mis en cause, en théorie, il semblerait que les Inuits «pourront être invités dans l'avenir à modifier l'application de ce droit pour tenir compte des activités touristiques» (Brooke, 2011 : 70). Il s'agira d'observer comment les populations locales gèreront cette incitation.

Cette réflexion nous amène à préciser ce que nous entendons par la participation des populations locales dans les projets de parcs nationaux. Cette participation se réalise principalement sur trois plans : l'élaboration du projet (limite, zonage, concept d'aménagement) dans le cadre d'un groupe de travail regroupant les organismes concernés, la consultation des populations locales lors des audiences publiques, la délégation de la gestion des parcs à un organisme ou une communauté autochtone, qui permettra la création d'emplois dont bénéficieront les populations locales.

La participation de la population se manifeste donc en amont de la création des parcs, dès l'amorce du projet, par la mise en place par le ministère du Développement durable, de l'Environnement et des Parcs (MDDEP) d'un groupe de travail comprenant tous les intervenants régionaux concernés. Par la suite, les populations des communautés concernées sont invitées à participer aux séances d'information et à ces audiences publiques, telles que celles qui se sont déroulées en avril et juin 2008 pour le projet de parc national Tursujuq (Adams, 2009 : 1). Parmi les demandes des participants aux audiences, soulignons que ces derniers appuient la demande des Cris et des Inuits afin qu'ils soient gestionnaires du parc, comme le prévoit le plan directeur provisoire (Adams, 2009 : 6 ). De plus, ils mentionnent que l'exploitation du parc, par sa création d'emplois, aura un effet positif sur les communautés locales, dont les jeunes (Adams, 2009 : 7). Ils notent que le parc de Pingualuit emploie 5 personnes à temps 
complet qui proviennent toutes de la communauté inuite de Kangiqsujuaq, de même que pour les 18 employés temporaires (idem). Ils souhaitent que le même modèle soit appliqué pour le parc national Tursujuq (idem). Les participants sont préoccupés par les sources de conflits qui pourraient émerger entre les visiteurs et les populations locales. Ils recommandent donc de suivre les orientations figurant à ce propos dans le Plan directeur provisoire au sujet de l'information à fournir aux visiteurs sur les activités traditionnelles de subsistance et d'ajuster l'offre des activités en fonction de ces dernières (Adams, 2009 : 8). Leur questionnement porte donc sur des éléments concrets liés à leur quotidien ainsi que sur les bénéfices qu'ils pourraient tirer de l'aménagement du parc.

Un autre aspect de la participation des communautés locales concerne la gestion des parcs. À ce propos, le gouvernement du Québec s'est engagé, en signant une entente de partenariat sur le développement économique et communautaire au Nunavik le 9 avril 2002, à ce que les Inuits participent à l'aménagement et à la gestion des parcs au Nunavik (MDDEP, 2007 : 31 ; BCDM Conseil Inc., 2008 : 21). Le gouvernement du Québec a délégué à l'Administration régionale Kativik (ARK) la gestion des parcs, entre autres pouvoirs (BCDM Conseil Inc., 2008 : 9). L'ARK est un organisme public créé en 1978. Il exerce sa compétence sur tout le territoire de Kativik comprenant l'ensemble des terres situées au nord du $55^{\mathrm{e}}$ parallèle, à l'exclusion de certaines terres attribuées aux Cris de la communauté de Whapmagoostui (BCDM Conseil Inc., 2006 : 1). Par ailleurs, l'ARK a des compétences en matière de formation et d'utilisation de la maind'œuvre sur son territoire. Pour cette raison, une entente a été conclue avec l'ARK. Ainsi, les gestionnaires des parcs sont appelés à se concerter avec les communautés concernées par l'entremise d'un comité d'harmonisation mis sur pied dès la création d'un parc. Sur le terrain, les résidents locaux sont favorisés à l'embauche, des entreprises locales sont appelées pour la gestion de certaines activités et certains services, les actions sont réalisées de façon concertée avec les organismes locaux, les associations locales participent au développement des parcs et une formation adaptée aux exigences des emplois offerts est fournie (MDDEP, 2007 : 31). De même, la participation des acteurs économiques régionaux fait partie intégrante de la stratégie touristique québécoise au nord du 49e parallèle (MTO, $2011: 10$ ).

Â ce jour, les populations locales sont conviées à la gestion des parcs en collaboration avec les autorités locales dans plusieurs projets de parcs. Par exemple, les Inuits sont sollicités pour leur connaissance des terrains du projet de parc national Ilittaniujalik (Brooke, 2011 : 71). Notons encore que, comme pour tous les projets de parc, un groupe de travail, composé entre autres de représentants de l'ARK, des villages d'Umiujaq, de Kuujjuarapik et de Whapmagoostui, a été mis en place pour la création du parc national Tursujuq (BCDM Conseil Inc., 2008 : 22). Depuis peu, le village d'Inukjuak est également associé au parc. De cette façon, le projet a été réalisé en collaboration étroite avec des personnes des trois communautés avoisinant le parc et a bénéficié de leurs connaissances. Nous observons le même type de participation pour l'élaboration du projet de parc national Kuururjuaq (entre autres des représentants de la municipalité de Kangiqsualujjuaq) (BCDM Conseil Inc., 2006 : 32). De même, la gestion et l'exploitation du parc national Albanel-Témiscamie-Otish seront confiées à la nation crie de Mistissini.

L'un des éléments majeurs découlant de la délégation de la gestion des parcs nationaux aux instances autochtones concerne le développement de la main-d'œuvre. En effet, l'aménagement des parcs puis leur exploitation demanderont l'engagement de personnes travaillant à temps complet ou pour des contrats saisonniers de trois à six mois. Tel sera le cas des communautés d'Umiujaq et de KuujjuarapikWhapmagoostui : 8 personnes embauchées pour 4,5 emplois à temps complet, ce qui représentera $26 \%$ de l'ensemble des emplois du parc (BCDM Conseil Inc., 2008 : vii). De même, pour le parc Kuururjuaq, il était prévu de créer 6 emplois à temps complet (soit 5 emplois à temps complet et 5 emplois saisonniers) pour la main-d'œuvre locale (BCDM Conseil Inc., 2006 : 3). La population de Kangiqsualujjuaq bénéficie de 6 emplois à temps complet (BCDM Conseil Inc., 2006 : 51), mais le projet ne précise pas de quels types de poste (accueil, guide, entretien, administration, etc.) il s'agit.

Même si le taux de chômage n'est pas mesuré officiellement ni en Jamésie, ni au Nunavik, ni dans les communautés locales, nous savons qu'il est plus élevé et que les variations mensuelles sont généralement plus hautes que pour l'ensemble du Québec (BCDM Conseil Inc., 2007 : 48; BCDM Conseil Inc., 2006 : 56-57). Nous pouvons toutefois présumer que les impacts de ces nouveaux emplois seront significatifs. En effet, les emplois créés par les parcs apporteront de l'argent frais dans la région. Dans le cas du parc national Albanel-Témiscamie-Otish, les revenus d'emplois du parc atteindront une moyenne par emploi de 51158 \$ (à savoir 2 emplois permanents à temps complet et 16 emplois saisonniers au bénéfice des communautés de Mistissini, Chapais, Chibougamau et Oujé-Bougoumou), ce qui est plus haut que le revenu annuel moyen de la région, soit 40864 \$ en 2004 (BCDM Conseil Inc., 2007 : 46-49), ceci sans compter les emplois provenant de l'aménagement du parc. Par ailleurs, dans le cas du parc Kuururjuaq, 36,8 \% des emplois totaux découlant de la gestion et de la fréquentation du parc seront attribués aux habitants du Nunavik, dont 11,3\% pour la communauté de Kangiqsualujjuaq (BCDM Conseil Inc., 2006 : 57-58). Notons que cette communauté bénéficiera des emplois les mieux rémunérés du parc, soit une moyenne s'élevant à 46964 \$ par an par emploi à temps complet (BCDM Conseil Inc., 2006 : 58).

Le cumul des effets de l'aménagement des quatre parcs du Nunavik cités en exemple aura donc un effet structurant sensible sur le plan économique pour l'ensemble de la région, que ce soit pour les retombées économiques transitoires ou permanentes. En outre, d'après une récente enquête sur le terrain, les employés inuits des parcs nationaux des Pingualuit et Kuururjuaq se disent heureux des emplois qu'ils ont obtenus dans les parcs (Brooke, $2011: 71$ ).

Comme les parcs nationaux comportent un volet éducatif, ils visent à favoriser la découverte du milieu par les touristes. Ainsi, les connaissances traditionnelles des communautés locales sont retenues et seront mises en valeur dans l'offre 
éducative. Le parc national Kuururjuaq comporte un volet éducatif auquel les Kangiqsualujjuamiuts participeront. En effet, le premier contact des visiteurs du parc se fera au village de Kangiqsualujjuaq, où a été développée une exposition permanente présentant les grands aspects et le patrimoine culturel du parc (MDDEP, $2007: 30$ ).

De même, les connaissances des aînés pourraient être mises à profit sur le plan de la culture et des activités de formation (MTO, 2011 : 17). La mise en valeur des cultures inuite et crie fait également partie du projet du futur parc national Tursujuq. Ainsi, la présence des deux peuples autochtones qui y sont établis sera mise à contribution (BCDM Conseil Inc., $2008: 11$ ).

Finalement, les populations locales profiteront directement du développement des parcs. En effet, il est prévu que les touristes passeront quelques jours dans les municipalités environnantes avant ou après leur séjour dans un parc national, ce qui engendrera des retombées directes et indirectes aux populations de ces municipalités. Selon une étude réalisée par la Sépaq, "pour 1 \$ dépensé dans un parc national, il y a $15 \$$ en termes de retombées économiques dans les villes et villages avoisinants» (Commission parlementaire, 2009). Dans le cas du projet de parc national Albanel-TémiscamieOtish, les retombées du parc profiteront principalement à la communauté crie de Mistissini, mais aussi aux municipalités de Chibougamau et de Chapais (Gouvernement du Québec, 2005 : 44).

En définitive, non seulement, la création des parcs nationaux préserve le patrimoine naturel et culturel et les met en valeur, mais elle bénéficie également aux économies locales (Brooke, 2011 : 70). En outre, en vertu des droits accordés aux Cris et aux Inuits, ceux-ci peuvent poursuivre leurs activités traditionnelles d'approvisionnement de chasse et pêche, ce qui est en soi une exception dans les parcs nationaux. Notons que les autres communautés locales ne bénéficient pas de cet avantage.

\section{Remerciements}

Nous remercions madame Nathalie Girard et monsieur Serge Alain du ministère du Développement durable, de l'Environnement, de la Faune et des Parcs, ainsi que madame Louise Séguin de Tourisme Québec, ministère des Finances et de l'Économie pour leur contribution au contenu de cette chronique.
Références

ADAMS, Johnny N. (2009) Projet de parc national des Lacs-GuillaumeDelisle-et-à-l'Eau-Claire. Rapport d'audiences, Ministère du Développement durable, de l'Environnement et des Parcs : Québec. $34 \mathrm{p}$.

BCDM Conseil Inc. (2006) Projet de parc national de la Kuururjuaq. Étude de retombées économiques, BCDM Conseil Inc. : Québec. 82 p.

BCDM Conseil Inc. (2007) Projet de parc national Albanel-TémiscamieOtish. Étude de retombées économiques, BCDM Conseil Inc. : Québec. $88 \mathrm{p}$.

BCDM Conseil Inc. (2008) Parc national des Lacs-Guillaume-Delisle-et-àl'Eau-Claire. Évaluation des retombées économiques, présenté à l'Administration régionale Kativik, BCDM Conseil Inc. : Québec. 110 p.

BROOKE, Lorraine (2011) Étude d'impact sur l'environnement et le milieu social. Projet de parc national des Monts-Pyramides, Ministère du développement durable, de l'environnement et des parcs (MDDEP) : Québec. 102 p.

Commission parlementaire (2009) «Les travaux parlementaires. Journal des débats. Étude des crédits du ministère du Développement durable, de l'Environnement et des Parcs (1) : volet Parcs. Le mardi 21 avril 2009 Vol. $41 \mathrm{~N}^{\circ} 3$ », Commission permanente des transports et de l'environnement, <http://www.assnat.qc.ca/Archives/fra/39Legislature1/ DEBATS/journal/cte/090421.htm\#_Toc228866105>, consulté le 4 juin 2012.

Gouvernement du Québec (2005) Projet de parc Albanel-Temiscamie-Otish E’weewach, Gouvernement du Québec: Québec. 92 p.

Ministère du développement durable, de l'environnement et des parcs (MDDEP) (2007) Plan directeur provisoire. Parc national de la Kuururjuaq, Gouvernement du Québec: Québec. 48 p.

Ministère du tourisme (MTO) (2011) Stratégie touristique québécoise au nord du $49^{\circ}$ parallèle. Cultures et espaces à découvrir, Gouvernement du Québec: Québec, 30 p.

QUÉBEC (1977, modifiée en 2001) Loi sur les parcs, L.R.Q., chapitre P-9, Québec : Éditeur officiel du Québec.

QUÉBEC (s. d.) Loi sur les droits de chasse et de pêche dans les territoires de la Baie James et du Nouveau-Québec, L.R.Q., chapitre D-13.1, Québec : Éditeur officiel du Québec. À jour au 1 1 mai 2012. 